Check for updates

Cite this: RSC Adv., 2017, 7, 35135

\title{
Characterization and acid-catalysed depolymerization of condensed tannins derived from larch bark
}

\author{
Aibin Zhang, ${ }^{a}$ Jiongjiong Li, (D) ${ }^{a}$ Shifeng Zhang, ${ }^{a}$ Youbing Mu, ${ }^{b}$ Wei Zhang ${ }^{\star a}$ \\ and Jianzhang Li $i^{\star a}$
}

Condensed tannins from larch bark extracts are a natural renewable and eco-friendly material and are potential substitutes for phenolic petrochemicals. However, the wide application of tannin is restricted by its low reactivity. Therefore, the goal of this study was to enhance the reactivity of larch tannin by depolymerization and determine optimal reaction conditions. The structures of larch tannin and depolymerized larch tannin were characterized by Fourier transform infrared (FT-IR) spectroscopy, solid phase ${ }^{13} \mathrm{C}-\mathrm{NMR}$ and matrix-assisted laser desorption ionization time-of-flight (MALDI-TOF) mass spectrometry. The thermal stability of larch tannin before and after depolymerization was evaluated by thermogravimetric analysis (TGA). The results indicated that the monomeric units of larch tannin were mainly composed of catechin/epicatechin, gallocatechin/epigallocatechin, catechin-gallocatechin esters, and stilbene glucosides. The presence of a catechin gallate dimer that had lost both gallic acid residues and a hydroxy group and a small amount of fisetinidin units were also observed. Additionally, a series of peaks corresponding to oligomers of larch tannin of up to 11 repeating units were observed from the MALDI-TOF MS data. Depolymerization treatment, especially using 2-mercaptoethanol as a nucleophilic reagent, was found to be beneficial to the enhancement of thermal stability. The optimization of the depolymerization reaction allowed the reaction to be completed in two hours, at $60{ }^{\circ} \mathrm{C}$, using 2-mercaptoethanol as a nucleophile and $0.1 \mathrm{~mol} \mathrm{~L}^{-1} \mathrm{HCl}$. Many compounds of molecular weight less than $600 \mathrm{Da}$, mainly dimers and monomers, were obtained under these reaction conditions.

Received 23rd March 2017 Accepted 4th July 2017

DOI: $10.1039 / \mathrm{c} 7 \mathrm{ra03410e}$

rsc.li/rsc-advances or urea with condensed tannins for the synthesis of bio-based epoxy resins, ${ }^{8-10}$ tannin-phenol-formaldehyde resins (TPF), ${ }^{\mathbf{1 1 - 1 3}}$ and tannin-urea-formaldehyde resins (TUF). ${ }^{\mathbf{1 4 , 1 5}}$ However, the polymeric forms of tannins are not as good as the monomers for use in the production of tannin-based resins because they react differently or lack sufficient reactivity., ${ }^{4,5,16}$ This is because macromolecular condensed tannins exhibit greater steric hindrance and the available reactive sites are far apart. ${ }^{\mathbf{1 7}, 18}$ Moreover, the increased rate of tannin substitution leads to decreased bond strength and increased viscosity of tanninbased resins, resulting in a low utilization rate of the condensed tannins. Therefore, the depolymerization of polyhydroxyflavan-3-ol polymers to oligomers or monomers is a promising approach to decrease steric hindrance and enhance chemical reactivity.

The depolymerization reaction of condensed tannins is usually achieved by acid-catalysed cleavage of the interflavan bonds in the presence of a nucleophilic reagent. ${ }^{19}$ Generally, the A-rings of all the flavan-3-ol subunits are protonated and the interflavan bonds are cracked, releasing terminal subunits as neutral monomeric flavan-3-ols and extension subunits as car-

bocations. The carbocations are trapped by the nucleophilic

\footnotetext{
${ }^{a}$ MOE Key Laboratory of Wooden Material Science and Application, Beijing Key Laboratory of Wood Science and Engineering, Beijing Forestry University, 35 Qinghua East Road, Haidian District, Beijing 100083, P. R. China. E-mail: zhangwei@bjfu.edu.cn; lijzh@bjfu.edu.cn; Tel: +86-10-62336912; +86-10-62338083

${ }^{b}$ CAS Key Laboratory of Bio-based Materials, Qingdao Institute of Bioenergy and Bioprocess Technology, Chinese Academy of Sciences, Qingdao 266101, China
} 


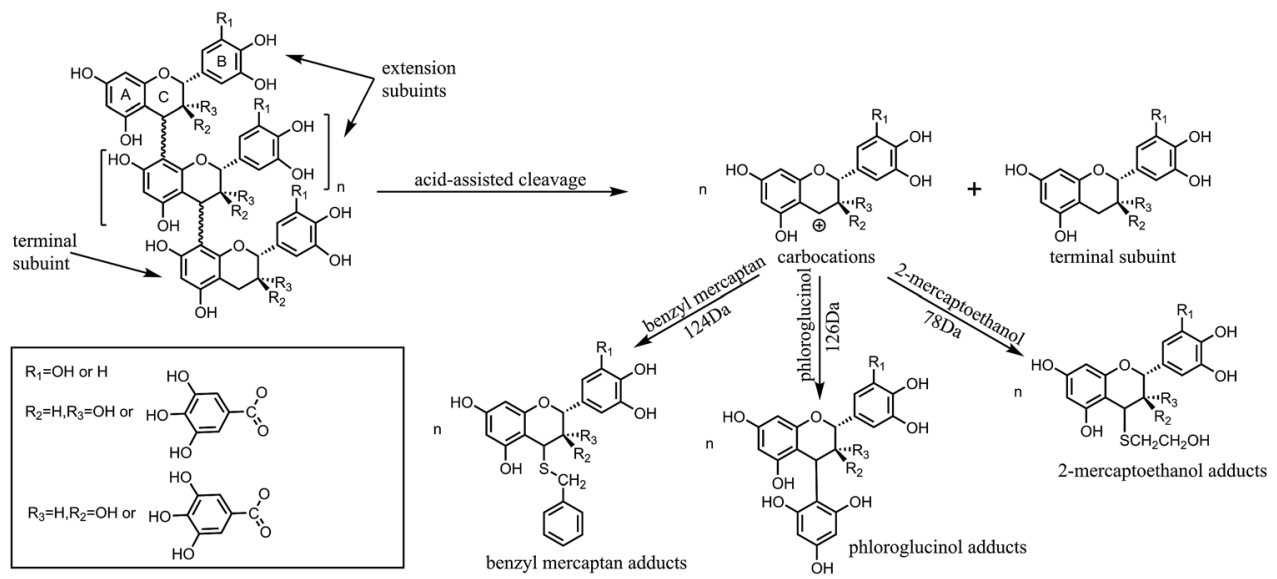

Fig. 1 Acid depolymerization of condensed tannins in the presence of nucleophiles.

reagent and transformed into the corresponding nucleophile adducts as shown in Fig. 1.

Different strategies have been developed for the depolymerization of condensed tannins in the presence of nucleophilic reagents, and the use of phloroglucinol, ${ }^{20}$ thioglycolic acid, ${ }^{21}$ benzyl mercaptan, ${ }^{22}$ benzene- $p$-sulfinic acid, benzenethiol $^{23}$ or 2-mercaptoethanol ${ }^{24}$ have already been reported. Among these nucleophiles, phloroglucinol and benzyl mercaptan are most commonly selected for the depolymerization of condensed tannins to determine the monomeric composition of tannins. ${ }^{20}$ Although good yields of monomeric phenolic synthons can be obtained with sulphur-containing compounds with unpleasant odor as nucleophilic reagents, the depolymerization process is also accompanied by the occurrence of some side reactions. Benzenethiol is easily oxidized in acidic medium and benzene- $p$-sulfinic acid has poor nucleophilicity for the depolymerization of polyphenols. ${ }^{23}$ Thioglycolic acid may polymerize into thioester and is unstable at room temperature. ${ }^{4}$ Therefore, compared to the other sulphurcontaining compounds, it seems less ideal to use the three nucleophiles for the depolymerization of condensed tannins. Moreover, the 2-mercaptoethanol odour is much less pronounced compared to that of thioglycolic esters and esterification side reaction cannot occur in the case of 2-mercaptoethanol. Phloroglucinol is odorless and therefore has no special handling requirements. In previous studies, (+)-catechin, $(-)$-epicatechin, or (-)-epigallocatechin gallate were used as chain breakers to depolymerize condensed tannins..$^{25,26}$ However, the goal of the present study is the production of phenolic synthons from renewable resources, so it seems inappropriate to use these monomers as nucleophiles. Hydrogen gas is another potential reducing agent that can be used for the depolymerization of tannins, but the hydrogenolysis reaction needs to be performed in a special reactor and the resulting yield of oligomers is low. ${ }^{5}$ Depolymerization under alkaline conditions is not ideal because of the competitive autoxidation of tannins and formation of catechinic acid, resulting in only a small percentage of condensed tannins being depolymerized. ${ }^{4,27}$ The effect of reaction conditions such as nucleophiles and temperature on the depolymerization of condensed tannins is significant. ${ }^{20,26,28}$ The depolymerization reaction is frequently applied to condensed tannins for structure elucidation, but studies of the optimal reaction conditions for the depolymerization of condensed tannins have been limited, and the yields for the conversion of condensed tannins into their constitutive subunits are low, with incomplete depolymerization. Therefore, to produce more monomers or oligomers, the conditions of the depolymerization reaction were investigated.

In this study, larch tannin (LT) (condensed tannins) extracted from larch bark was depolymerized to yield phenolic monomers. Mercaptoethanol (ML) was compared with benzyl mercaptan (BM) and phloroglucinol (PL) as depolymerization reagents for the depolymerization of larch tannin. The chemical structures of LT and depolymerized larch tannin (DLT) were detected using FT-IR, solid phase ${ }^{13} \mathrm{C}$-NMR and Matrix-assisted laser desorption ionization time-of-flight (MALDI-TOF) mass spectrometry. Thermal properties of the LT and DLT were characterized by thermogravimetric analysis (TGA). The depolymerization conditions including temperature, time, and catalyst concentration were optimized to enhance larch tannin reactivity to facilitate the development and utilization of larch tannin as potential substitutes of phenolic petrochemicals for bio-based polymers and materials.

\section{Experimental section}

\subsection{Materials}

Larch tannin was purchased from Tian'guan Biotech Co., Henan province, China. Phloroglucinol, benzyl mercaptan, 2mercaptoethanol and all other chemicals were obtained from Beijing Chemical Reagents Co., Ltd. (China). All chemicals were analytic grade and used without further purification.

\subsection{Optimization of the depolymerization reaction}

Using a final concentration of larch tannin of $24 \mathrm{mg} \mathrm{mL}^{-1}$, all the depolymerization reactions were conducted in a nitrogen atmosphere to prepare DLT products. In each run, larch tannin 
was dissolved in absolute ethanol to a concentration of $60 \mathrm{mg}$ $\mathrm{mL}^{-1}$, and $20 \mathrm{~mL}$ of the tannin stock solution was transferred to a $250 \mathrm{~mL}$ three-neck bottle loaded in a preheated water bath. To investigate the effect of nucleophiles on the depolymerization of tannins, different nucleophiles (5.2 $\mathrm{g}$ of phloroglucinol (PL), 4.9 $\mathrm{mL}$ of benzyl mercaptan (BM), or $2.9 \mathrm{~mL}$ of 2-mercaptoethanol (ML)) corresponding to 10 molar equivalents of catechin monomers were dissolved in $30 \mathrm{~mL}$ of absolute ethanol and injected into the $250 \mathrm{~mL}$ three-neck bottle. $414 \mu \mathrm{L}$ of $37 \% \mathrm{HCl}$ (i.e. $0.1 \mathrm{~mol} \mathrm{~L}^{-1} \mathrm{HCl}$ ) was applied and the reaction was performed at $60{ }^{\circ} \mathrm{C}$ under magnetic stirring at $500 \mathrm{rpm}$ for $2 \mathrm{~h}$. The obtained products were denoted as DLT-PL, DLT-BM, and DLTML, respectively.

In order to study the effect of reaction temperature on the depolymerization of tannins, $414 \mu \mathrm{L}$ of $37 \% \mathrm{HCl}$ and $2.9 \mathrm{~mL}$ of 2-mercaptoethanol as nucleophile were added to $30 \mathrm{~mL}$ of absolute ethanol. The reaction was carried out at 40,60 , or $80{ }^{\circ} \mathrm{C}$ under magnetic stirring at $500 \mathrm{rpm}$ for $2 \mathrm{~h}$. The samples obtained under different reaction temperatures were labeled DLT-ML 40, DLT-ML 60, and DLT-ML 80, respectively.

To investigate the effect of reaction time, the temperature was set at $60{ }^{\circ} \mathrm{C}$. The depolymerization reaction was performed 1,2 or $3 \mathrm{~h}$ under magnetic stirring at $500 \mathrm{rpm}$. In the reaction, $414 \mu \mathrm{L}$ of $37 \% \mathrm{HCl}$ was used as the catalyst and $2.9 \mathrm{~mL}$ of $2-$ mercaptoethanol was placed into $30 \mathrm{~mL}$ of absolute ethanol. The obtained products were labeled DLT-ML 1, DLT-ML 2, and DLT-ML 3, respectively.

To study the effect of catalyst content on depolymerization, 207,414 , or $828 \mu \mathrm{L}$ of $37 \% \mathrm{HCl}$ (i.e. 0.05 , 0.1 , or $0.2 \mathrm{~mol} \mathrm{~L}^{-1}$ $\mathrm{HCl}$, respectively) were added to $30 \mathrm{~mL}$ of absolute ethanol. The reaction was conducted under magnetic stirring at $500 \mathrm{rpm}$ for $2 \mathrm{~h}$ at $60{ }^{\circ} \mathrm{C}$, and with $2.9 \mathrm{~mL}$ of 2 -mercaptoethanol in the reactor. The samples obtained under different catalyst contents were denoted as DLT-ML 0.05, DLT-ML 0.1, and DLT-ML 0.2, respectively. When each reaction was complete, the resulting solutions in bottle were collected. The solutions were stored in a refrigerator before further processing and analysis.

\subsection{FTIR analysis}

The reaction products were freeze-dried by placing them in a vacuum freeze-drier (Sihuan LGJ-25C, Sihuan Co., Ltd, Beijing, China) for $48 \mathrm{~h}$. These freeze-dried samples were milled to 200 mesh meal and then recorded by FT-IR (Nicolet IS10, Thermo Fisher Scientific Corporation, Waltham, MA, USA) at a resolution of $4 \mathrm{~cm}^{-1}$. Each spectrum was obtained from 500 to $4000 \mathrm{~cm}^{-1}$ using 32 scans.

\subsection{Solid phase ${ }^{13} \mathrm{C}-\mathrm{NMR}$ analysis}

The solid-state ${ }^{13} \mathrm{C}$ CP-MAS NMR spectra of the freeze-dried samples were recorded on a Bruker 300 spectrometer at a frequency $75.47 \mathrm{MHz}$. Chemical shifts were calculated relative to TMS (tetramethylsilane). The rotor was spun at $12 \mathrm{kHz}$ on a double-bearing $5 \mathrm{~mm}$ Bruker probe.

\subsection{MALDI-TOF-MS analysis}

The MALDI-TOF mass spectra were performed on a MALDI-TOF instrument (AXIMA Performance, Shimadzu, Japan) equipped with a pulsed nitrogen laser (wavelength: $337 \mathrm{~nm}$, laser pulse length $3 \mathrm{~ns}$ ). The experimental conditions used were: polaritypositive, 100-150 pulses per spectrum, mass-high $(20 \mathrm{kV}$ acceleration voltage), and flight path-linear and 200-800 ns time of delayed extraction technique. Based on previous studies, the samples were first dissolved in acetone $(4 \mathrm{mg}$ $\mathrm{mL}^{-1}$ ). The sample solutions were mixed with an acetone solution consisting of 2,5-dihydroxy benzoic acid (DHB) as the matrix and acetone (10 $\mathrm{mg} \mathrm{mL}^{-1}$ acetone). For the enhancement of ion formation, $\mathrm{NaCl}$ was added to the matrix. The samples of the solution and matrix were mixed in equal amounts, and $0.5-1 \mu \mathrm{L}$ of the resulting mixture was placed on the MALDI target. The solvent was evaporated on the MALDI target before placing into the spectrometer. ${ }^{14,29}$

\subsection{Thermogravimetric analysis (TGA)}

Thermal decomposition was performed with a Q50 TGA analyzer (TA instruments, USA). To do this, $5-8 \mathrm{mg}$ of freezedried sample was transferred to a platinum pan and the temperature ranged from 25 to $600{ }^{\circ} \mathrm{C}$ at a heating rate of $10{ }^{\circ} \mathrm{C}$ $\min ^{-1}$ in a nitrogen atmosphere $\left(40 \mathrm{~mL} \mathrm{~min}{ }^{-1}\right)$. The curves of the percentage of weight loss and the first time derivative were determined using the TGA software.

\section{Results and discussion}

\subsection{Characterization of larch tannin}

3.1.1. FT-IR analysis of larch tannin. The FTIR spectrum of larch tannin in the range from 400 to $4000 \mathrm{~cm}^{-1}$ is shown in Fig. 2. The broad peaks in the region $3000-3500 \mathrm{~cm}^{-1}$ were assigned to $-\mathrm{OH}$ stretching vibration. The peaks near $2931 \mathrm{~cm}^{-1}$ were due to $\mathrm{C}-\mathrm{H}$ stretching vibration assigned to $-\mathrm{CH}$ and $-\mathrm{CH}_{2}$ groups of the aliphatic hydrocarbon. ${ }^{30,31}$ This band originates from - $\mathrm{CH}$ stretch vibration in aromatic methoxyl groups and in the methyl and methylene groups of side chains. Aromatic and nonaromatic $\mathrm{C}-\mathrm{H}$ bond deformation appeared near 3000 $\mathrm{cm}^{-1} .{ }^{32,33}$ The absorption band at $1704 \mathrm{~cm}^{-1}$ was attributed to

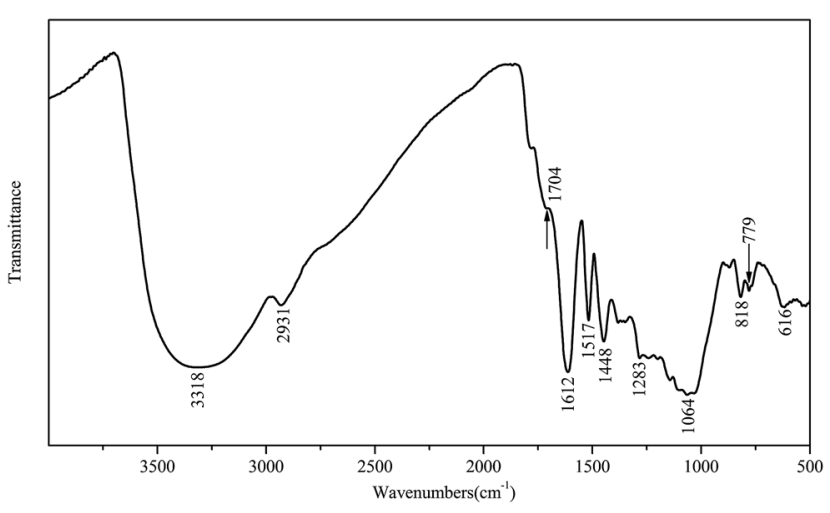

Fig. 2 FT-IR spectrum of larch tannin. 
the carboxylic acid $-\mathrm{C}=\mathrm{O}$ of the galloyl unit on the epicatechin gallate. ${ }^{34,35}$ The bands between $1610 \mathrm{~cm}^{-1}$ and $1445 \mathrm{~cm}^{-1}$ were attributed to aromatic ring stretching vibration. These aromatic rings are no changes to the "core" of the tannin structure during the depolymerization procedures. The single peak at $1517 \mathrm{~cm}^{-1}$ indicated that the larch tannin predominantly consisted of procyanidin ${ }^{36}$ and the high intensity of the $\mathrm{C}-\mathrm{C}$ stretching peak at $1612 \mathrm{~cm}^{-1}$ indicated the presence of a high number of $\mathrm{C} 4-\mathrm{C} 8$ interflavonoid linkages. ${ }^{35}$ The bands observed from 1283 to $1060 \mathrm{~cm}^{-1}$ were the characteristic bands of $\mathrm{C}-\mathrm{O}-\mathrm{C}$ including aromatic $\mathrm{C}-\mathrm{O}$ and aliphatic $\mathrm{C}-\mathrm{O}$, and bands in the range of $890-$ $680 \mathrm{~cm}^{-1}$ were associated with $\mathrm{C}-\mathrm{H}$ of benzene rings and $\mathrm{O}-\mathrm{H}$ of alcohol vibration. ${ }^{37}$

3.1.2. ${ }^{13} \mathrm{C}-\mathrm{NMR}$ analysis of larch tannin. The solid state ${ }^{13} \mathrm{C}$ NMR spectrum of larch tannin (Fig. 3) was interpreted according to the previous studies. ${ }^{35,38-40}$ From the spectrum of larch tannin, the peak at $175 \mathrm{ppm}$ represented the carbonyl group $\mathrm{C}=\mathrm{O}$ of the gallic residue linked in $\mathrm{C} 3$ to the heterocycle ring of a flavonoid structure. The band of high intensity at $154 \mathrm{ppm}$ was assigned to $\mathrm{C} 5, \mathrm{C} 7$ and $\mathrm{C} 9$ of the aromatic A-ring. The band at $143 \mathrm{ppm}$ was attributed to the $\mathrm{C} 3^{\prime}$ and $\mathrm{C}^{\prime}$. The split peak at 143 and $154 \mathrm{ppm}$ (phenolic peak) showed the characteristic of tannins. The signals at 129 and $116 \mathrm{ppm}$ can be respectively assigned to the $\mathrm{C}^{\prime}{ }^{\prime}$ and $\mathrm{C}^{\prime}{ }^{\prime} \mathrm{B}$ ring sites. The peak at $116 \mathrm{ppm}$ showed that larch tannin consists of both pyrogallol and catechol B-rings with the latter being more predominant. This was consistent with the finding from FT-IR analysis that larch tannin is predominantly consisted of procyanidin.

The band at $104 \mathrm{ppm}$ was assigned to the interflavonoid bonds $\mathrm{C} 4-\mathrm{C} 8$ and $\mathrm{C} 4-\mathrm{C} 6$. The absence of the $\mathrm{C} 4-\mathrm{C} 6$ interflavonoid band at $95 \mathrm{ppm}$ could indicate that the predominant interflavonoid linkage was $\mathrm{C} 4-\mathrm{C} 8$, which was in accordance with the FTIR analysis that showed high number of C4-C8 linkages. The low band intensity at $98 \mathrm{ppm}$ was corresponded to C6, C8 and C10. Peaks between $60 \mathrm{ppm}$ and $85 \mathrm{ppm}$ were assignable to carbohydrates present in the tannin. Resonance at $71 \mathrm{ppm}$ could correspond to C3 in the chain interior and upper chain-ending positions. Its higher intensity could be due to the

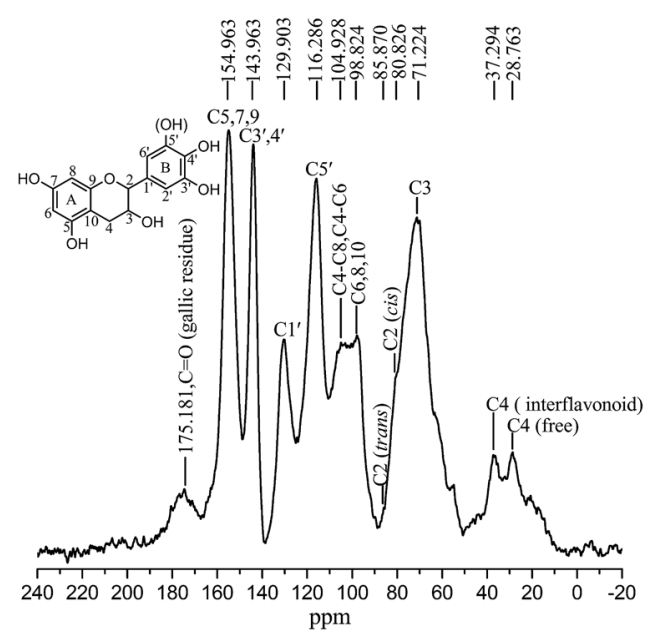

Fig. 3 Solid-state ${ }^{13} \mathrm{C}$ CPMAS NMR spectrum of the larch tannin. superposition of the oligomeric carbohydrates in the larch tannin. The peaks for $\mathrm{C} 2$ were covered by the $\mathrm{C} 3$ band but were visible as shoulder of the latter, especially the $\mathrm{C} 2$ (cis) at $80 \mathrm{ppm}$ and C2 (trans) at $85 \mathrm{ppm}$. C4 of expansion units showed a peak at $37 \mathrm{ppm}$, while the terminal $\mathrm{C} 4$ showed a characteristic chemical shift at $28 \mathrm{ppm}$.

3.1.3. MALDI-TOF-MS analysis of larch tannin. Larch tannin extracted from larch bark is a kind of condensed tannin generally composed of flavan-3-ol repeating units (such as procyanidin, prodelphinidin, prorobinetinidin, and profisetinidin), smaller fractions of simple sugars, and other polysaccharides. ${ }^{14}$ Fig. 4 shows the MALDI-TOF spectra of larch tannin.

The spectra indicated that the identical larch tannin monomer constituents in accordance with the previous data were galloyl group, (epi)catechin, (epi)gallocatechin, and (epi)gallocatechin gallate with molecular weights (MW), respectively, of $152 \mathrm{Da}, 290 \mathrm{Da}, 306 \mathrm{Da}$, and $458 \mathrm{Da}$ (not marked in Fig. 4b) (Fig. 5). ${ }^{14} \mathrm{~A}$ shift of approximately 440 Da among the peaks (e.g. $1610.5-1171.5=439 \mathrm{Da}, 1874.7-1436.5=438.2 \mathrm{Da})$ was
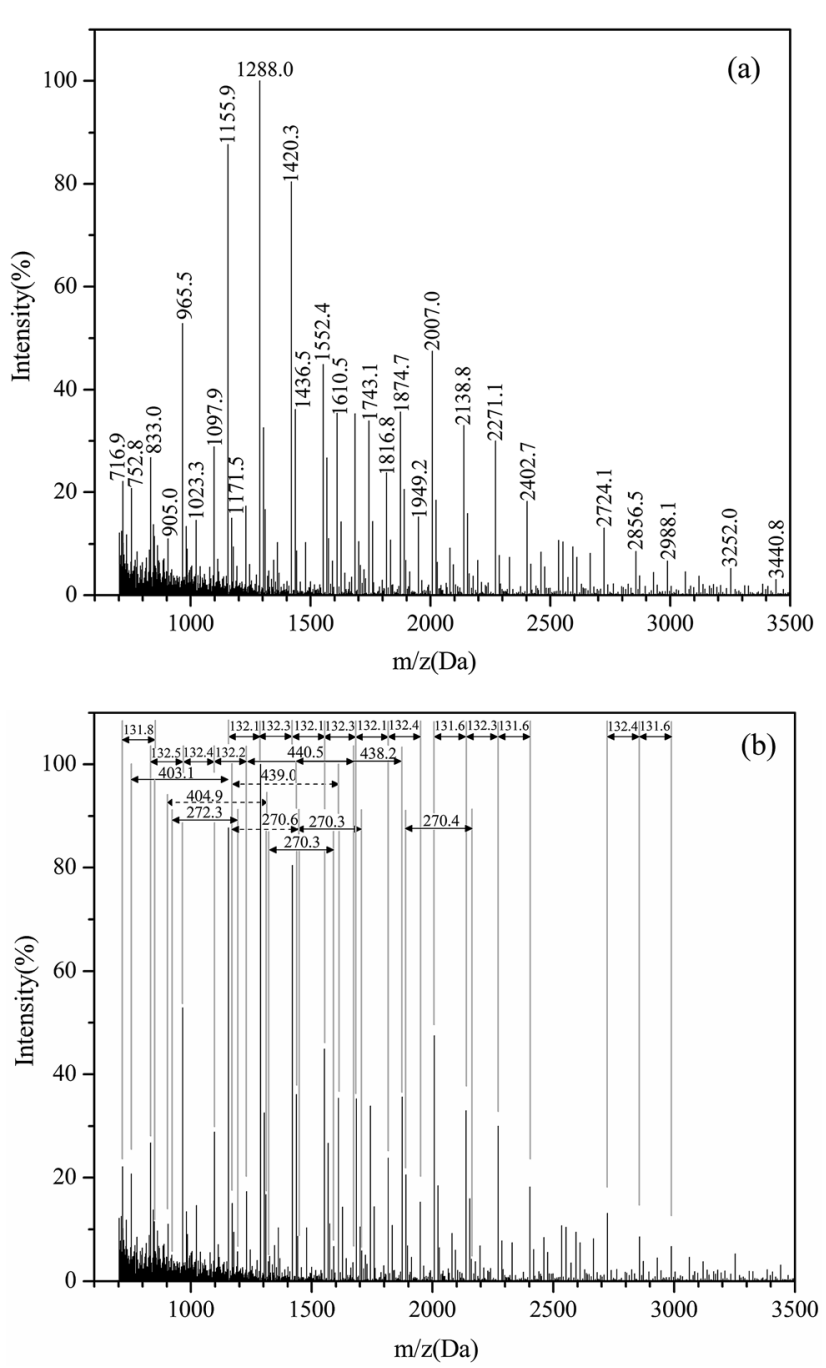

Fig. 4 The MALDI-TOF spectra of (a) larch tannin and (b) indication of the relevant $132 \mathrm{Da}, 272 \mathrm{Da}, 404 \mathrm{Da}$, and $440 \mathrm{Da}$ repeat units. 
<smiles>Oc1cc(O)c2c(c1)OC(c1ccc(O)c(O)c1)C(O)C2</smiles>

(epi)catechin<smiles>CC(=O)c1cc(O)c(O)c(O)c1</smiles>

galloyl group

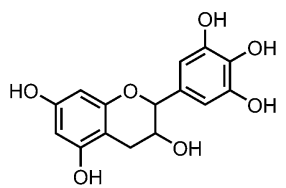

(epi)gallocatechin

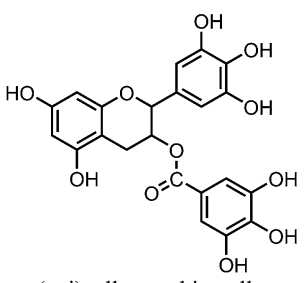

(epi)gallocatechin gallate
Fig. 5 Molecular structures of the most common flavan-3-ols units in larch tannin.

observed in the mass spectra. This mass change was associated with the loss of a (epi)catechin gallate unit $(442.4-2 \mathrm{H}=440.4$ Da) (Fig. 6a). ${ }^{29,38}$ The peak at 905.0 Da was interpreted as a (epi) catechin gallate dimer $\left(2 \times(442.4-1 \mathrm{H})+\mathrm{Na}^{+}=905.8 \mathrm{Da}\right)$. The mass spectra indicated clearly that a shift of molecular mass of about 404 Da was observed, equal to the mass of stilbene glucoside astringin (Fig. 6b) when covalently bonded within a condensed tannin molecule $(406.4-2 \mathrm{H}=404.4 \mathrm{Da}) .{ }^{\mathbf{4 1 , 4 2}}$ The peak at 833.0 Da may reflect an astringin dimer $(2 \times(406.4-$ $\left.1 \mathrm{H})+\mathrm{Na}^{+}=833.8 \mathrm{Da}\right)$. There was therefore a reasonable possibility of astringin units as part of the building blocks of the larch tannin. The presence of stilbenes and their glucosides in the molecular structure of bark tannins from European larch was suggested previously. ${ }^{42}$ However, there was only a low amount of astringin units as indicated by the very low intensity of the peaks. More detailed analyses should be carried out to confirm the structure. Some low intensity peaks with mass increments of about 272 Da indicated that tetrahydroxy flavan units were possibly present in the structure of larch tannin (Fig. 6c). This unit could be formed by (epi)catechin gallate units in which the gallic acid residue has been removed. ${ }^{38}$ The mass spectra indicated that a main repetition unit of approximately 132 Da was observed, but no known flavonoid structure<smiles>O=C(OC1Cc2c(O)cc(O)cc2OC1c1ccc(O)c(O)c1)c1cc(O)c(O)c(O)c1</smiles><smiles>Oc1cc(O)c2c(c1)OC(c1ccc(O)c(O)c1)CC2</smiles>

(c)

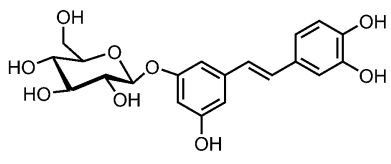

(b)

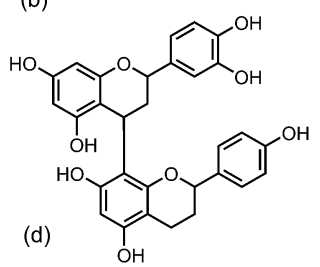

Fig. 6 The monomeric structures in larch tannin. corresponds to that molecular weight. In previous studies, a similar mass spectrum for larch tannin was reported and suggested that the repeating unit is not $132 \mathrm{Da}$ but appeared to be $528 \mathrm{Da}(=4 \times 132 \mathrm{Da})$ (Fig. $6 \mathrm{~d})$. The repeating unit is a dimer of structure $\mathrm{C}$ (Fig. 6c) with a $-\mathrm{OH}$ group missing. The unit has alcoholic hydroxyl groups that were lost at the separation of the gallic acid and a single phenolic hydroxyl group was also lost. $^{38,42}$ These unusual structures were different from the commonly reported flavan-3-ols. Larch tannin with different polymerization degrees exhibited chemical structures that consisted of flavan-3-ol units and non-flavan-3-ol units, mainly including carbohydrates, ${ }^{42}$ which is in accordance with NMR analysis. Unfortunately, some peaks were not explained by structural units described above, suggesting that larch tannin had other monomer structures in addition to the observed structures. However, many different structural units in condensed tannins caused relevant MALDI peaks as reported by other researchers. ${ }^{29,39}$ Additionally, the MALDI-TOF spectra clearly showed different polymer chain length distributions of the larch tannin up to 11 repeating units.

3.1.4. Thermogravimetric analysis of larch tannin. Fig. 7 shows the TGA and derivative thermal degradation (DTG) profiles of larch tannin. The TGA-DTG thermograms of larch tannin revealed two obvious mass losses. First, there was an initial weight loss that could be ascribed to the loss of adsorbed water. The second loss, that is more important, was due to larch tannin decomposition. The degradation onset temperature of larch tannin was $170{ }^{\circ} \mathrm{C}$, evidence of purity, since tannins with abundant amounts of carbohydrate exhibit a lower degradation onset temperature compared to extracts that are relatively free of carbohydrate. ${ }^{31,43}$ The data indicated the presence of sugar moieties in larch tannin structures and some easily-degraded sugars, consistent with that of MALDI-TOF-MS analysis. Two decomposition peaks appeared at $233{ }^{\circ} \mathrm{C}$ and $266{ }^{\circ} \mathrm{C}$ in the second weight loss stage with a residue rate of $50.4 \%$ obtained at the end of the decomposition process. The larch tannin was thermally more stable than other condensed tannins analyzed under the same conditions in other studies, namely the

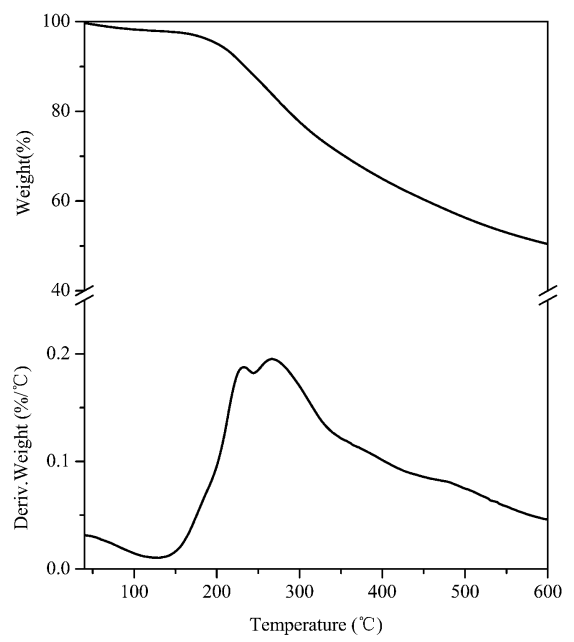

Fig. 7 TG and DTG curves of larch tannin. 
quebracho tannins and mimosa tannins that decompose at $150{ }^{\circ} \mathrm{C}$ and $146{ }^{\circ} \mathrm{C}$, respectively. ${ }^{40,44}$

\subsection{Optimization of depolymerization process}

3.2.1. Impact of the nucleophile. The solution of larch tannin in ethanol was depolymerized using phloroglucinol, benzyl mercaptan, or 2-mercaptoethanol as nucleophiles at $60{ }^{\circ} \mathrm{C}$ for $2 \mathrm{~h}$ with $414 \mu \mathrm{L}$ of $37 \% \mathrm{HCl}$. Fig. 8 shows the FT-IR spectra of DLT and PL in the range from 400 to $4000 \mathrm{~cm}^{-1}$. Some peaks of these spectra were consistent with those of larch tannin. The weak bands at $699 \mathrm{~cm}^{-1}$ and $668 \mathrm{~cm}^{-1}$ were attributed to $\mathrm{C}-\mathrm{S}$ bond. ${ }^{45,46}$ These bands were not observed in the spectrum of LT but were present in the spectra of DLT-BM and DLT-ML. The presence of these bands confirmed that the mercapto group was added to the flavonoid structure of larch tannin, indicating that the larch tannin was successfully depolymerized and the thiol-nucleophiles had a positive effect on the depolymerization of larch tannin. The peaks of DLT-PL spectrum were obviously different in the spectra of DLT-BM and DLT-ML, and extremely similar to the spectrum of PL due to the presence of high amounts of phloroglucinol in the DLT-PL, implying a minor effect of phloroglucinol on the depolymerization of larch tannin under these reaction conditions. From the FT-IR analysis, we concluded that benzyl mercaptan and 2mercaptoethanol were promising depolymerization reagents for use in the degradation of larch tannin.

The solid state ${ }^{13} \mathrm{C}$ NMR spectra of DLT-BM, DLT-PL and DLT-ML are shown in the Fig. 9. From Fig. 9a, the appearance of the new peaks, at $137 \mathrm{ppm}, 134 \mathrm{ppm}$ and $39 \mathrm{ppm}$ in the case of DLT-BM compared to LT, was assignable to C of benzyl SH ring and $\mathrm{C} 4-\mathrm{SCH} 2$, respectively. ${ }^{47,48}$ The peaks at 128 ppm for DLT$\mathrm{BM}$ and $129 \mathrm{ppm}$ for LT were assigned to $\mathrm{C}^{\prime}$. The increasing intensity of the $\mathrm{C}^{\prime}$ peak of DLT-BM may be caused by the introduction of benzyl $\mathrm{SH}$ ring into the structure of tannin. These results illustrated the occurrence of the reaction between larch tannin and benzyl mercaptane. The depolymerization of tannins is usually achieved by acid-catalysed cleavage of the interflavan bonds $\mathrm{C} 4-\mathrm{C} 8$ and $\mathrm{C} 4-\mathrm{C} 6$ in the presence of

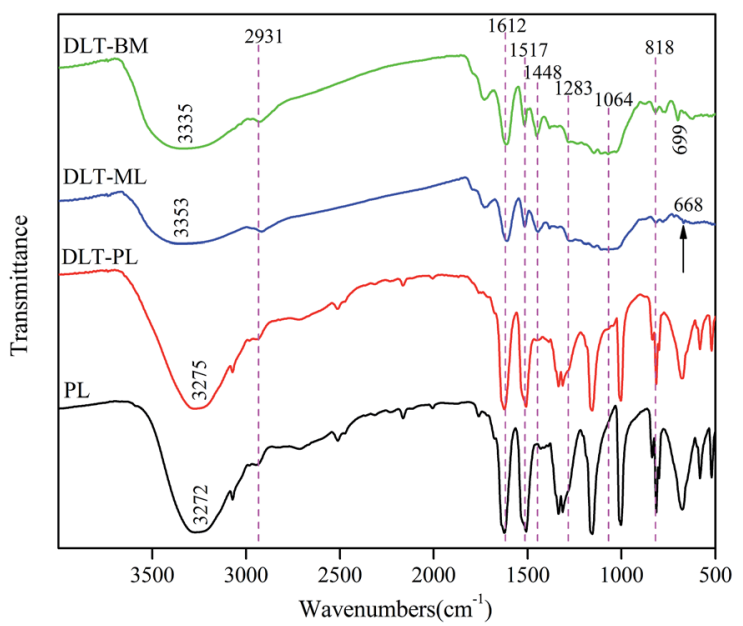

Fig. 8 FT-IR spectra of DLT and PL.
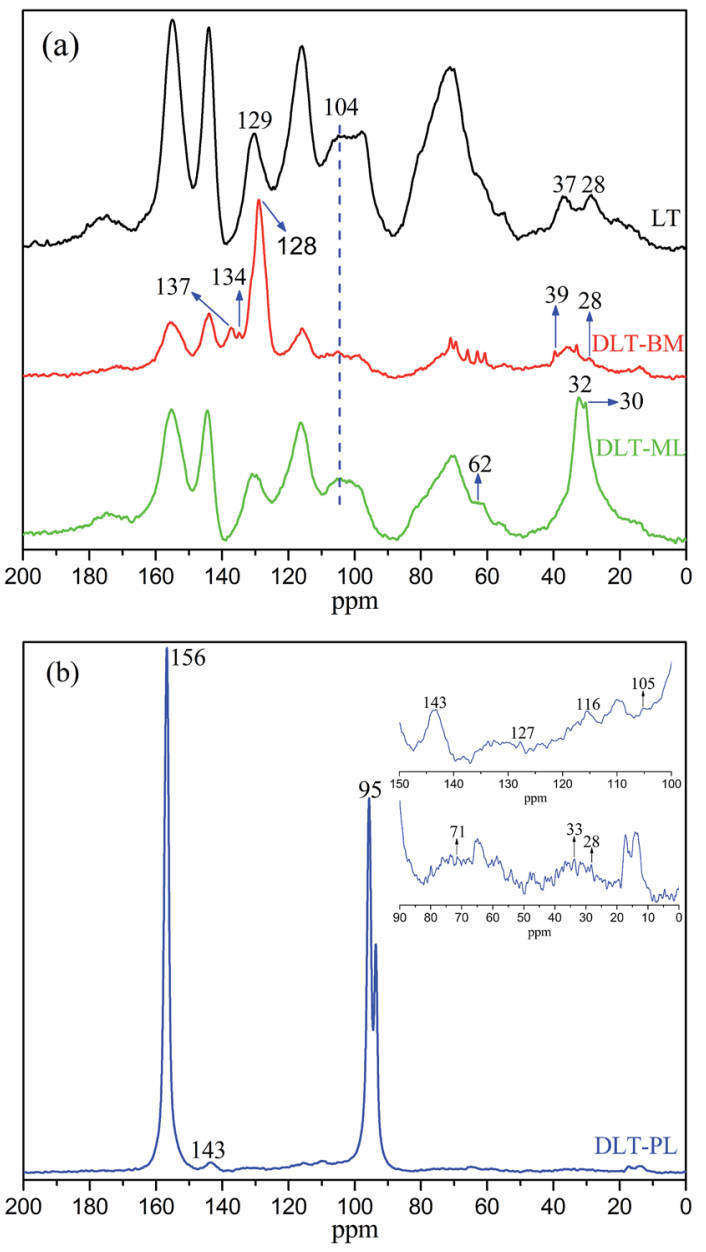

Fig. 9 The solid-state ${ }^{13} \mathrm{C}$ CPMAS NMR spectra of larch tannin depolymerized in the presence of different nucleophiles. (a) DLT-BM and DLT-ML, and (b) DLT-PL.

a nucleophilic reagent. The $\mathrm{C} 4$ sites in the form of carbocations react with nucleophilic reagents. The intensity of the broad peak, around $104 \mathrm{ppm}$ in the spectra of DLT-BM and DLT-ML, was largely weakened compared to that of LT. This peak was attributed to the interflavan bonds $\mathrm{C} 4-\mathrm{C} 8$ and $\mathrm{C} 4-\mathrm{C} 6$, and the weakening of its intensity indicated that great amounts of the interflavan bonds have been cleaved and turned into $-\mathrm{C}-\mathrm{S}-$ groups. The band at $62 \mathrm{ppm}$ was attributed to $\mathrm{CH}_{2} \mathrm{OH}$ of the mercaptoethanol. ${ }^{\mathbf{4 9 , 5 0}}$ The peak at $37 \mathrm{ppm}$, which corresponds to interflavan C4, disappeared in the spectrum of the DLT-ML, and inversely, the higher intensity new peak at 32 ppm was obtained. These results indicated that larch tannin was successfully depolymerized by 2-mercaptoethanol and interflavan bonds were converted to $\mathrm{C}-\mathrm{S}$ bonds.

From Fig. 9b, the two dominant peaks at $156 \mathrm{ppm}$ and $95 \mathrm{ppm}$ were assignable to the larch tannin and phloroglucinol carbons. The band at 105 ppm was due to the site of phloroglucinol connecting with the $\mathrm{C} 4$ of the tannin units, and the $\mathrm{C} 4$ was shown by the band at $33 \mathrm{ppm} .^{20}$ These results indicated that larch tannin reacted with phloroglucinol under acidic condition. Based on the above analysis, the monomeric units, mainly 
catechin, epicatechin and flavanol-3-O-gallate, oligomers and corresponding derivatives co-existed in the larch tannin depolymerized by different nucleophiles, which are consistent with previous studies. The peak intensity at $30 \mathrm{ppm}$ in NMR spectrum of DLT-ML was stronger than that of the DLT-BM and DLT$\mathrm{PL}$ at $28 \mathrm{ppm}$, and the intensity of these peaks related to free C4 was weaker in the NMR spectra of the DLT-BM and DLT-PL than that of DLT-ML. These results indicated that 2-mercaptoethanol was the best nucleophile for the depolymerization of larch tannin.

The MALDI-TOF spectra were used to describe the molecular weight change of larch tannin depolymerized in the presence of different nucleophiles (Fig. 10). Most of the reaction product major peaks were differed those of the larch tannin. After depolymerization, the area of polymer peaks decreased and conversely, many low molecular weight peaks appeared in these spectra but were not present in the spectrum of larch tannin, suggesting that interflavan bonds were successfully broken down and the depolymerization of larch tannin was feasible under such reaction conditions. In Fig. 10a, several series with a repeating interval of $124 \mathrm{Da}$ were noted in the DLT-BM spectra, the DLT-PL spectra displayed a main repetition unit of $125 \mathrm{Da}$, and DLT-ML samples showed a repetition unit of about $77 \mathrm{Da}$. These masses may be associated with a benzyl mercaptan unit $(124-1 \mathrm{H}=123 \mathrm{Da})$, a phloroglucinol unit (126 $-1 \mathrm{H}=125 \mathrm{Da})$, and a mercaptoethanol unit $(78-1 \mathrm{H}=77 \mathrm{Da})$, respectively. Therefore, the three kinds of nucleophiles were successfully added to flavonoid structures, consistent with the FT-IR and NMR results. The mass spectra of 200 to $1000 \mathrm{Da}$ clearly indicated dominant fragments at $391.7 \mathrm{Da}(290.3+23+$ $78=391.3 \mathrm{Da})$ for DLT-BM, 455.7 Da $(378.4+78=456.4 \mathrm{Da})$ for DLT-PL, and 311.2 Da $(290.3+23=313.3 \mathrm{Da})$ for DLT-ML (not marked in Fig. 10a), which may be due to a higher ratio of these peaks corresponding to the depolymerization products observed for these samples. As it can be seen from the spectra, the peaks sometimes did not include the sodium ion. The intense peaks of the mass spectra of the three samples were obviously different in the range from 500 to $3500 \mathrm{Da}$ (Fig. 10b). The mass spectra of the DLT-BM and DLT-PL showed higher molecular mass peaks than those of DLT-ML. The mass spectra displayed the maximal molecular weight fragmentation peaks obtained at 1557.2 Da (pentamer) for DLT-BM, 1786.3 Da (hexamer) for DLT-PL and 1125.6 Da (trimer or tetramer) for DLT-ML. The DLT-ML exhibited the lowest polymerization degree followed by DLT-BM and DLT-PL, which was explained by the different nucleophilicity of different nucleophilic reagents in the acidified ethanol solution. In the depolymerization reaction, the difficulty is not the bond-breaking process of the interflavan $\mathrm{C}-\mathrm{C}$ bonds, which can be readily cleaved under "mild" acidic conditions, but the trapping of the resulting carbocations. This indicates that the nucleophilicity of nucleophilic reagents is the key to the depolymerization reaction. Additionally, in the presence of flanking hydroxy groups at both the 3-and 5-positions, the bulky phloroglucinol and benzyl mercaptan cannot easily approach the carbocations. ${ }^{23}$ Therefore, we concluded that the 2-mercaptoethanol had better nucleophilicity than the two other reagents. These results were
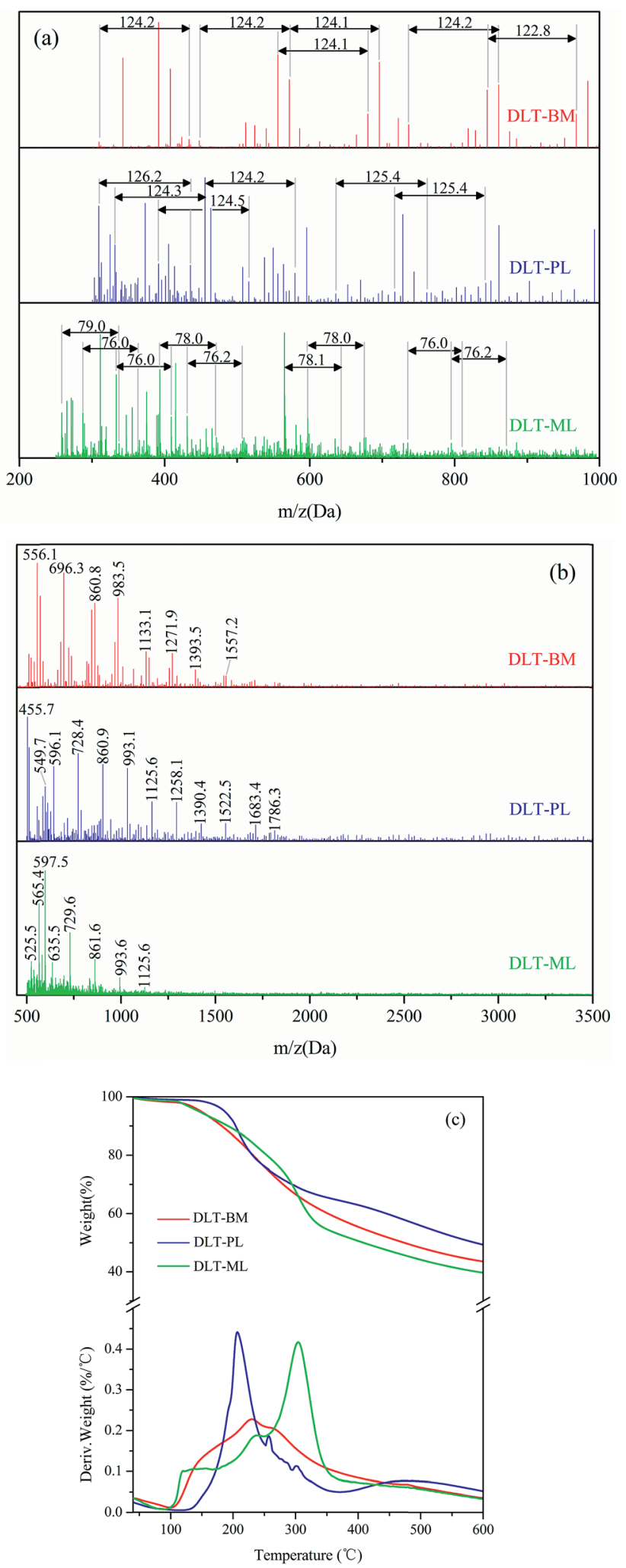

Fig. 10 The MALDI-TOF spectra of larch tannin depolymerized in the presence of different nucleophiles. (a) 200-1000 Da, (b) 500-3500 $\mathrm{Da}$, and (c) thermogravimetric degradation curves and the first derivatives of DLT-BM, DLT-PL, and DLT-ML. 
consistent with a previous report that benzyl mercaptan is a better reagent than phloroglucinol for the depolymerization reaction of tannins. ${ }^{20}$ Moreover, it can be concluded that the monomers, oligomers and corresponding nucleophile adducts co-existed in the reaction solutions from the MALDI-TOF spectra. These products with high reactivity are suitable as the substitutes of phenolic petrochemicals for bio-based polymers and materials, especially the resins and foams. ${ }^{51}$

The TGA-DTG curves of DLT-BM, DLT-PL, and DLT-ML are shown in Fig. 10c. Under nitrogen atmosphere, the three DLT samples started to decompose at $160{ }^{\circ} \mathrm{C}$ for DLT-PL, $149{ }^{\circ} \mathrm{C}$ for DLT-BM and $140{ }^{\circ} \mathrm{C}$ for DLT-ML, which showed lower initial thermal stability than LT. Thermal degradation profiles of DLTBM and DLT-ML revealed that the two samples had relatively lower decomposition onset than DLT-PL. This difference was due to the formation of carbon-sulfur bonds at the pyranyl ring (4-position) during the depolymerization reaction that promoted thermal instability. Similarly, the presence of a sulfonate group at the 4 position promoted thermal instability. ${ }^{52}$ In the second loss stage, the first decomposition peak of the DLT-PL and DLT-BM was slightly lower than that of the LT, while the first decomposition temperature of DLT-ML at $239^{\circ} \mathrm{C}$ was higher than that of the LT and showed better thermal stability. Compared to the DLT-ML and DLT-BM, DLT-PL (207 ${ }^{\circ} \mathrm{C}$ ) showed a low decomposition peak and exhibited the worst thermal stability in the second stage, which may be due to the decomposition of phloroglucinol as discussed in the FT-IR analysis section. For the DLT-BM, the shape of the curve and the position of the degradation steps were similar to those of the LT. The second decomposition peak of DLT-ML at the second weight loss stage at $304{ }^{\circ} \mathrm{C}$ was higher than that of LT, which indicated that the DLT-ML samples exhibited better thermal resistance than the LT. This indicates that the larch tannin was depolymerized into small molecules showing better thermal stability than the polymers, and no molecular bonds between the monomer structures of larch tannin were broken during degradation. The two distinct degradation peaks of DLTML were similar to those of catechin at $230{ }^{\circ} \mathrm{C}$ and $310^{\circ} \mathrm{C}$ in the second stage, ${ }^{52}$ revealing that DLT-ML samples were composed of more monomers or oligomers. The TGA-DTG analysis clearly indicated that the thermal stability of depolymerized larch tannin was enhanced and the DLT-ML samples exhibited better thermal stability than LT and other depolymerization products. Based on the analysis data, we concluded that 2-mercaptoethanol was the best nucleophile for the depolymerization of larch tannin.

3.2.2. Impact of reaction temperature. We next investigated the effect of reaction temperature on the process. To do this, the depolymerization of larch tannin using 2-mercaptoethanol as the nucleophile was carried out at $40{ }^{\circ} \mathrm{C}, 60{ }^{\circ} \mathrm{C}$, and $80{ }^{\circ} \mathrm{C}$ for $2 \mathrm{~h}$ with $414 \mu \mathrm{L}$ of $37 \% \mathrm{HCl}$. The MALDI-TOF-MS spectra obtained from larch tannin depolymerized at varying temperatures are shown in Fig. 11. In the range from 200 to $1000 \mathrm{Da}$, the three reaction products major peaks obtained under our conditions were mostly similar, with dominant peaks at 311.2 Da and 338.4 Da $(258.3+78=336.3 \mathrm{Da})$, and maximal peaks at 993.3 $\mathrm{Da}, 967.2 \mathrm{Da}$, and $923.2 \mathrm{Da}$, respectively, for the
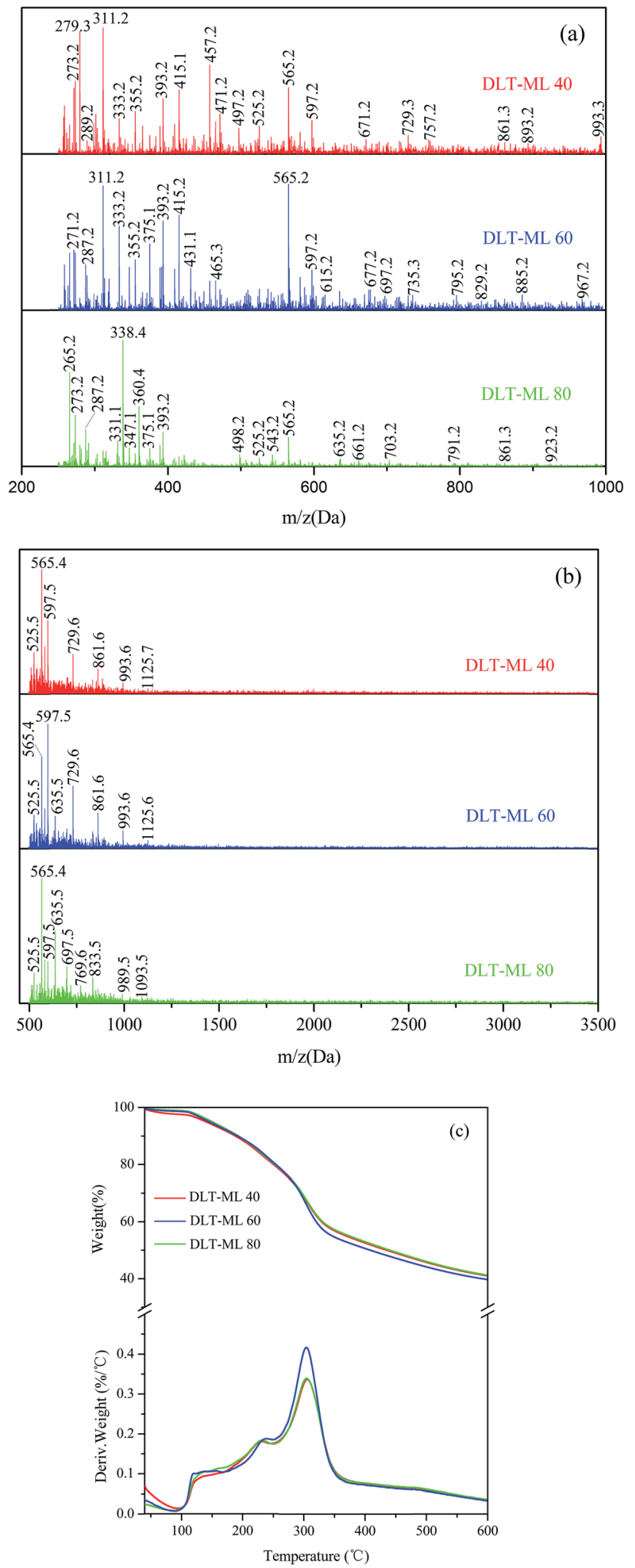

Fig. 11 The MALDI-TOF spectra of larch tannin depolymerized with varying temperatures. (a) 200-1000 Da, (b) 500-3500 Da, and (c) thermogravimetric degradation curves and the first derivatives of DLTML prepared at different temperatures. 
trimer. In terms of mass distribution, the three DLT-ML mass spectra showed no obvious differences in the range from 500 to $3500 \mathrm{Da}$. There was high relative intensity (100\%) for the DLTML 40 and DLT-ML 80 at peak 565.4 Da (dimer), but the relative intensity (100\%) peak of DLT-ML prepared at $60{ }^{\circ} \mathrm{C}$ was at 597.5 Da (dimer). A considerable amount of oligomers, especially those with molecular weights of 565.4 Da and 597.5 Da, were present in these DLT-ML samples obtained at different temperatures. The same lowest peaks in the mass spectra of the three reaction products were detected at 525.5 Da and were assigned to the dimer. High molecular weight fragmentation peaks were obtained at 1125.7 Da for DLT-ML 40, 1125.6 Da (trimer or tetramer) for DLT-ML 60, and 1093.5 Da (trimer) for DLT-ML 80. The spectra of the larch tannin depolymerized by 2mercaptoethanol at different temperatures showed different polymer chain length distributions varying from monomers to trimers. However, the causal mechanism of this effect remained unclear. Indeed, the flavanol autoxidation and condensation that occur under acidic conditions could promote both intermolecular and intramolecular reactions and the formation of other types of tannin structures, some that are resistant to depolymerization..$^{\mathbf{2 3 , 5 3 , 5 4}}$ The flavanol autoxidation process is also susceptible to the influence of reaction conditions (e.g. temperature, $\mathrm{pH}){ }^{55,56}$ Therefore, different reaction conditions may influence the depolymerization reaction by affecting the flavanol autoxidation process, resulting in different experimental results.

The thermal curves of larch tannin depolymerized by 2mercaptoethanol at different temperatures are shown in Fig. 11c. A similar degradation thermogram was observed for the three DLT-ML samples prepared at different temperatures. In the second degradation step, the first degradation peaks were observed at $231{ }^{\circ} \mathrm{C}, 239{ }^{\circ} \mathrm{C}$, and $229^{\circ} \mathrm{C}$ for DLT-ML 40, DLT-ML 60 and DLT-ML 80, respectively, and the second degradation peaks were fairly similarly positioned at around $304{ }^{\circ} \mathrm{C}$. Additionally, the char yields obtained at $600{ }^{\circ} \mathrm{C}$ were about $41 \%$ for the three samples. The thermal decomposition behavior of DLTML 60 was dramatically decreased after $300{ }^{\circ} \mathrm{C}$, reaching to the same level as that of the DLT-ML 40 and DLT-ML 80. The depolymerization temperature affected the thermal decomposition behavior of the DLT-ML samples prepared at different temperatures mainly in the low temperature range of 100$250{ }^{\circ} \mathrm{C}$. The DLT-ML 60 showed a slightly higher thermal stability in comparison with DLT-ML 40 and DLT-ML 80 in the range of $100-250{ }^{\circ} \mathrm{C}$. The curves of the depolymerized larch tannin at different temperatures indicated that the optimal depolymerization temperature of larch tannin was $60{ }^{\circ} \mathrm{C}$.

3.2.3. Impact of reaction time. Fig. 12 show the MALDITOF mass spectra of larch tannin after depolymerization using 2-mercaptoethanol as the nucleophile at $60{ }^{\circ} \mathrm{C}$ for 1,2 , or $3 \mathrm{~h}$ with $414 \mu \mathrm{L}$ of $37 \% \mathrm{HCl}$. Most of major peaks of the DLT-ML samples were similar but their intensity was different between 200 and 1000 Da. The MALDI-TOF spectra clearly showed dominant fragment peaks at $311 \mathrm{Da}$ and $565 \mathrm{Da}$, and the presence of different oligomers with a polymerization degree of up to 3 units. From 500 to $3500 \mathrm{Da}$, there were obvious differences between the three mass spectra, with the appearance of
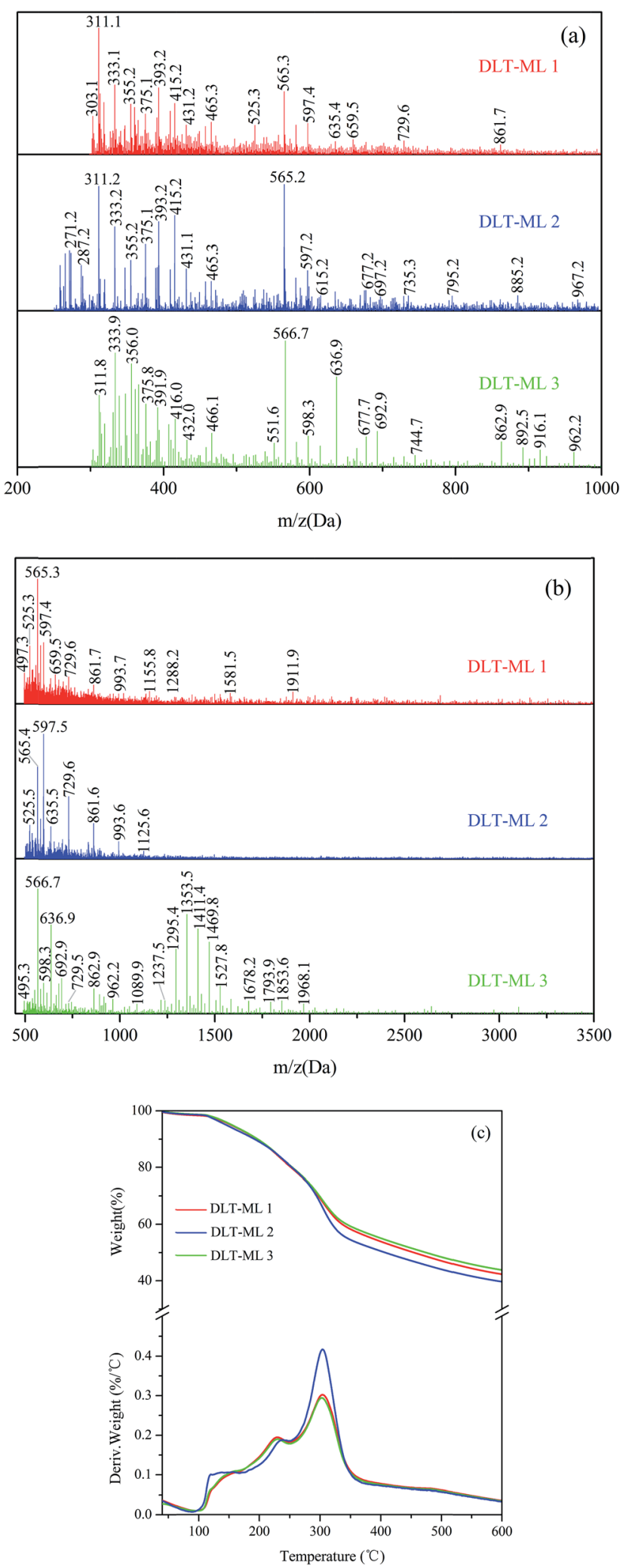

Fig. 12 The MALDI-TOF spectra of larch tannin depolymerized with varying amounts of time. (a) 200-1000 Da, (b) 500-3500 Da, and (c) thermogravimetric degradation curves and the first derivatives of DLTML prepared under different amounts of time. 
new high molecular mass peaks for DLT-ML 1 and DLT-ML 3. These new high mass peaks were different from those of the larch tannin, which may be due to condensation and the rearrangement of molecules in the depolymerization process. A sample possessing oligomers up to 6 repeating units was obtained based on the 1911.9 Da peak observed in the spectrum of DLT-ML 1. Analysis of DLT-ML 3 showed that the sample with more high molecular mass peaks was present in the form of oligomers up to 7 repeating units. It can be concluded that the degree of polymerization values decreased initially and then increased along with the reaction time from 1 to $3 \mathrm{~h}$, indicating a more pronounced effect of reaction time on larch tannin depolymerization.

Shown in Fig. 12c are the thermal degradation profiles of depolymerized larch tannin for 1, 2, or $3 \mathrm{~h}$. During degradation, the two-step degradation thermograms of the DLT-ML samples prepared for different times were extremely similar, especially the second degradation peaks at $c a .304{ }^{\circ} \mathrm{C}$. The thermal degradation of DLT-ML 2 was faster than that of the other two samples at temperatures higher than $250{ }^{\circ} \mathrm{C}$. In the second degradation step, the first maximum degradation temperature for the samples subjected to depolymerization for $1 \mathrm{~h}, 2 \mathrm{~h}$, or $3 \mathrm{~h}$ were $229^{\circ} \mathrm{C}, 239{ }^{\circ} \mathrm{C}$ and $230{ }^{\circ} \mathrm{C}$, respectively, indicating higher thermal stability for DLT-ML 2. However, higher residual mass percentage values at $600{ }^{\circ} \mathrm{C}$ were observed for DLT-ML $1(42.3 \%)$ and DLT-ML 3 (43.8\%), and the char yield for DLT-ML 2 was $39.7 \%$. Based on the above analysis, we can easily conclude that the larch tannin was optimally depolymerized for $2 \mathrm{~h}$.

3.2.4. Impact of catalyst concentration. DLT-ML samples were obtained using 2-mercaptoethanol as the nucleophile at $60{ }^{\circ} \mathrm{C}$ for $2 \mathrm{~h}$ with varying amounts of $\mathrm{HCl}$ as catalyst $(0.05-$ $0.2 \mathrm{~mol} \mathrm{~L}^{-1}$ ). Fig. 13 show the MALDI-TOF mass spectra of the DLT-ML samples obtained with different catalyst concentrations. The dominant peaks of DLT-ML 0.1 were $311.2 \mathrm{Da}$ and 565.2 Da in the range from 200 to $1000 \mathrm{Da}$. Compared to DLTML 0.1 , the high relative intensity $(100 \%)$ peaks at $393.0 \mathrm{Da}$ for DLT-ML 0.05 and DLT-ML 0.2 were identical. This value was described by the expression $M=23(\mathrm{Na})+290.3+78$ (2-
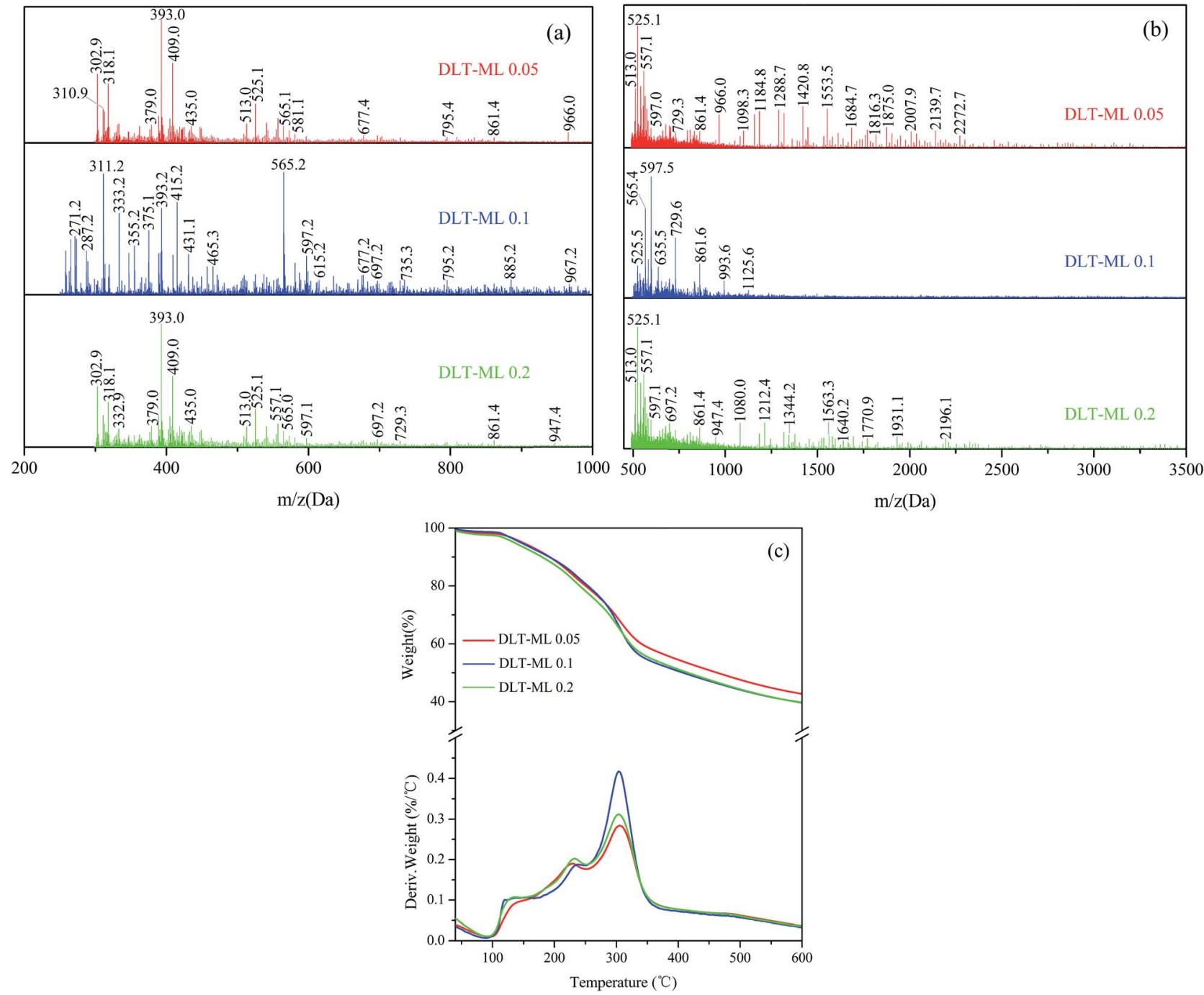

Fig. 13 The MALDI-TOF spectra of larch tannin depolymerized with varying catalyst concentrations. (a) 200-1000 Da, (b) 500-3500 Da, and (c) thermogravimetric degradation curves and the first derivatives of DLT-ML prepared with different catalyst concentrations. 
mercaptoethanol). The maximum peaks for the three DLT-ML samples prepared with different catalyst concentrations were 966.0 Da, 967.2 $\mathrm{Da}$, and 947.4 $\mathrm{Da}$, respectively. The relative intensity of these maximum peaks was weak, which indicated that the amounts of maximum peaks corresponding to those oligomers were very small. In the range from 500 to $3500 \mathrm{Da}$, new high molecular mass peaks were observed from the mass spectra of the prepared DLT-ML 0.05 and DLT-ML 0.2. Most of mass peaks of the DLT-ML 0.05 were the same as those of larch tannin, indicating that larch tannin was ineffectively depolymerized into oligomers at this catalyst concentration. The maximum peaks were at 2272.7 Da for DLT-ML 0.05 and 2196.1 Da for DLT-ML 0.2, respectively, corresponding to a flavonoid octamer and a flavonoid heptamer. Based on the maximum peaks, the degree of polymerization for DLT-ML 0.05 and DLTML 0.2 was higher than that of DLT-ML 0.1. Additionally, the presence of intense peaks in the lower mass range for the three DLT-ML samples indicated the presence of a considerable amount of compounds of molecular weight below $600 \mathrm{Da}$. The oligomers, especially dimers, were the most abundant, consistent with the high relative intensity $(100 \%)$ peaks at $525.1 \mathrm{Da}$ and 597.5 Da. Similarly, there was noticeable effect of catalyst concentration on the depolymerization of larch tannin.

The TGA-DTG curves of depolymerized larch tannin using 2mercaptoethanol as nucleophile with different catalyst concentrations are shown in Fig. 13c. The first degradation peak $\left(232{ }^{\circ} \mathrm{C}\right)$ of DLT-ML 0.2 in the second thermal event was higher than that of the DLT-ML $0.05\left(227^{\circ} \mathrm{C}\right)$. Compared to the DLT-ML 0.2 , the DLT-ML 0.1 had a higher degradation temperature (239 ${ }^{\circ} \mathrm{C}$ ) and showed better thermal stability. The second degradation peaks were fairly similarly positioned at around $304{ }^{\circ} \mathrm{C}$ for the three samples. The thermal degradation of the DLT-ML 0.1 and DLT-ML 0.2 was faster than that of DLT-ML 0.05 at temperatures above $250{ }^{\circ} \mathrm{C}$. The residual mass percentage values obtained at $600{ }^{\circ} \mathrm{C}$ were about $39 \%$ for the DLT-ML 0.1 and DLT-ML 0.2, slightly lower than that of DLT-ML 0.05. From the MALDI-TOF-MS and TGA-DTG analysis, these results indicated that $0.1 \mathrm{~mol} \mathrm{~L}^{-1} \mathrm{HCl}$ was the optimal catalyst concentration for the depolymerization of larch tannin.

\section{Conclusions}

The condensed tannins extracted from larch tannin with polymer chain lengths up to 11 units were mainly composed of procyanidins, prodelphinidins and flavan-3-ol gallates. The presence of the stilbene glucosides and small proportion of fisetinidin units was also suggested. Some oligomers appeared to contain a catechin gallate dimer that has lost both the gallic acid residues and a hydroxygroup at their terminal unit. These peculiar structures of larch tannin may affect behavior in resin production or as heavy metal scavengers. Thermogravimetric analysis revealed higher thermal stability of larch tannin compared to quebracho and mimosa tannins. Larch tannin was successfully depolymerized into monomers and/or oligomers under the acidic condition by different nucleophiles. The degradation products obtained using 2-mercaptoethanol as a nucleophilic reagent displayed better thermal stability than the un-depolymerized larch tannin. Furthermore, 2-mercaptoethanol was very effective in the depolymerization process of larch tannin. The optimized reaction conditions allowed the efficient depolymerization of larch tannin into monomers and/ or oligomers at $60^{\circ} \mathrm{C}$, for $2 \mathrm{~h}$, using 2-mercaptoethanol as the nucleophile, with $0.1 \mathrm{~mol} \mathrm{~L}^{-1} \mathrm{HCl}$. A high amount of the dimers and monomers were obtained under these optimized reaction conditions. Depolymerization of larch tannin was expected to increase their reactivity.

These results provide a promising method to obtain biobased phenolic monomers and the possibility for further high value-added utilization of larch tannin as the substitutes of phenolic petrochemicals for bio-based polymers and materials, especially the resins and foams.

\section{Acknowledgements}

This research was supported by the Fundamental Research Funds for the Central Universities (No. 2016ZCQ01), the CAS Key Laboratory of Bio-based Materials (No. KLBM2016002), and the Chinese National Science and Technology Support Program (2015BAD14B0302).

\section{References}

1 J. L. Yuan, Z. H. Gao and X. M. Wang, Pigm. Resin Technol., 2009, 38, 290-297.

2 P. J. Hernes and J. I. Hedges, Anal. Chem., 2000, 72, 51155124.

3 P. J. Hernes and J. I. Hedges, Geochim. Cosmochim. Acta, 2004, 68, 1293-1307.

4 L. Roumeas, C. Aouf, E. Dubreucq and H. Fulcrand, Green Chem., 2013, 15, 3268-3275.

5 Z. Li, J. J. Zeng, Z. H. Tong, Y. J. Qi and L. W. Gu, Food Chem., 2015, 188, 337-342.

6 C. N. Aguilar and G. Gutiérrez-Sánchez, Food Sci. Technol. Int., 2001, 7, 373-382.

7 L. Wang, W. Y. Liang, J. Yu, Z. X. Liang, L. L. Ruan and Y. C. Zhang, Environ. Sci. Technol., 2013, 47, 5771-5777.

8 C. Aouf, C. Le Guernevé, S. Caillol and H. Fulcrand, Tetrahedron, 2013, 69, 1345-1353.

9 H. Nouailhas, C. Aouf, C. Le Guerneve, S. Caillol, B. Boutevin and H. Fulcrand, J. Polym. Sci., Part A: Polym. Chem., 2011, 49, 2261-2270.

10 S. Benyahya, C. Aouf, S. Caillol, B. Boutevin, J. P. Pascault and H. Fulcrand, Ind. Crops Prod., 2014, 53, 296-307.

11 S. Jahanshaei, T. Tabarsa and J. Asghari, Pigm. Resin Technol., 2012, 41, 296-301.

12 A. Moubarik, A. Pizzi, A. Allal, F. Charrier and B. Charrier, Ind. Crops Prod., 2009, 30, 188-193.

13 E. C. Ramires and E. Frollini, Composites, Part B, 2012, 43, 2851-2860.

14 J. Z. Zhang, H. J. Kang, Q. Gao, J. Z. Li, A. Pizzi and L. Delmotte, J. Appl. Polym. Sci., 2014, 131, 547-557.

15 M. Zanetti, V. Causin, R. Saini, A. Cardin and R. Cavalli, Eur. J. Wood Wood Prod., 2014, 72, 385-392. 
16 S. Deprez, I. Mila, J.-F. Huneau, D. Tome and A. Scalbert, Antioxid. Redox Signaling, 2001, 3, 957-967.

17 A. P. Barbosa, E. B. Mano and C. T. Andrade, For. Prod. J., 2000, 50, 89-92.

18 A. Pizzi, J. Appl. Polym. Sci., 1979, 24, 1257-1268.

19 S. Matthews, I. Mila, A. Scalbert, B. Pollet, C. Lapierre, C. L. M. Hervé du Penhoat, C. Rolando and D. M. X. Donnelly, J. Agric. Food Chem., 1997, 45, 1195-1201.

20 J. A. Kennedy and G. P. Jones, J. Agric. Food Chem., 2001, 49, 1740-1746.

21 L. Mouls and H. Fulcrand, Tetrahedron, 2015, 71, 3012-3019.

22 J. Rigaud, J. Perez-Ilzarbe, J. M. R. Da Silva and V. Cheynier, J. Chromatogr. A, 1991, 540, 401-405.

23 B. R. Brown and M. R. Shaw, J. Chem. Soc., Perkin Trans. 1, 1974, 2036-2049.

24 W. Chen, C. L. Fu, Y. Qin and D. J. Huang, Food Chem., 2009, 114, 874-880.

25 T. Esatbeyoglu, V. Wray and P. Winterhalter, J. Agric. Food Chem., 2010, 58, 7820-7830.

26 H. W. Liu, T. Zou, J. M. Gao and L. W. Gu, Food Chem., 2013, 141, 488-494.

27 B. L. White, L. R. Howard and R. L. Prior, J. Agric. Food Chem., 2010, 58, 7572-7579.

28 L. W. Gu, M. A. Kelm, J. F. Hammerstone, G. Beecher, J. Holden, D. Haytowitz and R. L. Prior, J. Agric. Food Chem., 2003, 51, 7513-7521.

29 C. W. Oo, A. Pizzi, H. Pasch and M. J. Kassim, J. Appl. Polym. Sci., 2008, 109, 963-967.

30 W. J. Lee and W. C. Lan, Bioresour. Technol., 2006, 97, 257264.

31 S. Ben Mahmoud, H. Saad, B. Charrier, A. Pizzi, K. Rode, N. Ayed and F. Charrier-El Bouhtoury, Wood Sci. Technol., 2014, 49, 205-221.

32 R. Soto, J. Freer and J. Baeza, Bioresour. Technol., 2005, 96, 95-101.

33 L. Chupin, C. Motillon, F. C.-E. Bouhtoury, A. Pizzi and B. Charrier, Ind. Crops Prod., 2013, 49, 897-903.

34 Z. H. Huang, B. Zhang and G. Z. Fang, BioResources, 2013, 8, 4593-4608.

35 C. W. Oo, M. J. Kassim and A. Pizzi, Ind. Crops Prod., 2009, 30, 152-161.

36 L. Y. Foo, Phytochemistry, 1981, 20, 1397-1402.

37 R. C. Zhou, S. X. Si and Q. Y. Zhang, Appl. Surf. Sci., 2012, 258, 3578-3583.
38 P. Navarrete, A. Pizzi, H. Pasch, K. Rode and L. Delmotte, Ind. Crops Prod., 2010, 32, 105-110.

39 M. B. Ucar, G. Ucar, A. Pizzi and O. Gonultas, Ind. Crops Prod., 2013, 49, 697-704.

$40 \mathrm{H}$. Saad, A. Khoukh, N. Ayed, B. Charrier and F. C.-E. Bouhtoury, Ind. Crops Prod., 2014, 61, 517-525.

41 S. Bianchi, A. N. Gloess, I. Kroslakova, I. Mayer and F. Pichelin, Ind. Crops Prod., 2014, 61, 430-437.

42 S. Bianchi, I. Kroslakova, R. Janzon, I. Mayer, B. Saake and F. Pichelin, Phytochemistry, 2015, 120, 53-61.

43 A. Duval and L. Averous, J. Agric. Food Chem., 2016, 64, 17511760.

44 C. H. Luo, W. Grigsby, N. Edmonds, A. Easteal and J. AlHakkak, J. Appl. Polym. Sci., 2010, 117, 352-360.

45 Y. Sert, A. A. El-Emam, O. A. Al-Deeb, A. A. Al-Turkistani, F. Ucun and C. Cirak, Spectrochim. Acta, Part A, 2014, 126, 86-97.

46 F. Hassan, A. A. Hameed, A. Alshanon, B. M. Abdullah, H. Z. Huri, N. Hairunisa and E. Yousif, Asian J. Biochem., 2015, 10, 252-266.

47 S. Sivakumaran, A. L. Molan, L. P. Meagher, B. Kolb, L. Y. Foo, G. A. Lane, G. A. Attwood, K. Fraser and M. Tavendale, Phytochemistry, 2004, 65, 2485-2497.

48 K. Kamiya, C. Watanabe, H. Endang, M. Umar and T. Satake, Chem. Pharm. Bull., 2001, 49, 551-557.

49 Y. Matsuo, Y. Fujita, S. Ohnishi, T. Tanaka, H. Hirabaru, T. Kai, H. Sakaida, S. Nishizono and I. Kouno, Food Chem., 2010, 121, 1073-1079.

50 R. Kusano, S. Ogawa, Y. Matsuo, T. Tanaka, Y. Yazaki and I. Kouno, J. Nat. Prod., 2010, 74, 119-128.

51 A. Arbenz and L. Avérous, Green Chem., 2015, 17, 2626-2646. 52 M. Gaugler and W. J. Grigsby, J. Wood Chem. Technol., 2009, 29, 305-321.

53 A. Vernhet, S. Dubascoux, B. Cabane, H. Fulcrand, E. Dubreucq and C. Poncet-Legrand, Anal. Bioanal. Chem., 2011, 401, 1559-1569.

54 L. Mouls and H. Fulcrand, J. Mass Spectrom., 2012, 47, 14501457.

55 H. Fulcrand, M. Dueñas, E. Salas and V. Cheynier, Am. J. Enol. Vitic., 2006, 57, 289-297.

56 H. Fulcrand, V. Atanasova, E. Salas and V. Cheynier, The Fate of Anthocyanins in Wine: Are There Determining Factors? In Red Wine: Color-Revealing the Mysteries, ed. A. L. Waterhouse and J. A. Kennedy, American Chemical Society, Washington, DC, 2004, vol. 6, pp. 68-88. 